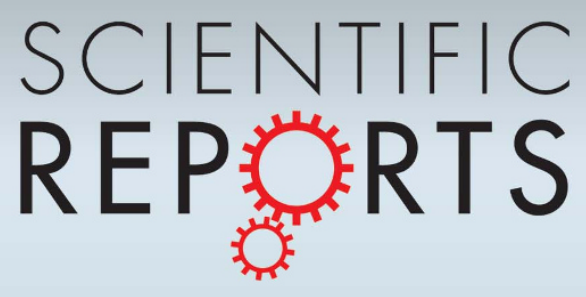

OPEN

SUBJECT AREAS:

PARASITOLOGY

BIOPHYSICS

COMPUTATIONAL BIOLOGY

STRUCTURAL BIOLOGY

Received

12 September 2011

Accepted

17 November 2011

Published

1 December 2011

Correspondence and requests for materials should be addressed to

A.S. (amit.icgeb@ gmail.com)

\section{Structural insights into thioredoxin-2: a component of malaria parasite protein secretion machinery}

\author{
Ashwani Sharma, Arvind Sharma, Sameer Dixit \& Amit Sharma
}

Structural and Computational Biology Group, International Centre for Genetic Engineering and Biotechnology (ICGEB), Aruna Asaf Ali Road, New Delhi, 110067, India.

Thioredoxins are vital components of Plasmodium proteome and act as both reducing agents and protein disulfide reductases. The malaria parasite $P$. falciparum thioredoxin-2 ( $P f \operatorname{Trx}-2)$ is part of the multi-protein complex embedded within the parasite parasitophorous vacuolar membrane (PVM) which purportedly directs protein secretion. We have characterized structural and enzymatic features of PfTrx-2, and we show that PfTrx-2 adopts a canonical thioredoxin fold but with significant structural differences in its $\mathrm{N}$-terminus. Our confocal localization data suggest distinct PVM residency of PfTrx-2. Based on the crystal structure of PfTrx-2, we screened and tested small molecule drug-like libraries for compounds which target unique structural features of PfTrx-2. Disruption of PfTrx-2 interactions using specific inhibitors may result in a dysfunctional parasite translocon that is rendered unable to secrete pathogenic proteins into hosts. This approach therefore offers a new focus for anti-malarial drug development.

M alaria is one of the world's most important infectious diseases and is responsible for enormous mortality and morbidity ${ }^{1}$. The unicellular parasite Plasmodium falciparum causes most aggressive form of this disease, leading to severe malaria ${ }^{1}$. Malaria parasites are constantly exposed to high fluxes of toxic reactive oxygen species (ROS) and to counter this they have developed elaborate redox systems comprising of thioredoxins, peroxoredoxins, glutathiones and superoxide dismutases amongst others ${ }^{2}$. Maintenance of proper redox balance is therefore clearly vital for erythrocytic stages of Plasmodium, as has been demonstrated by malaria protective effects of glucose-6-phosphate deficiency ${ }^{3}$. A tight control on parasitic redox balance is also important due to the different intra- and extracellular environments the parasite is exposed to. Oxidative stress is induced within the parasite due to its high metabolic and replicative rates, due to the constant reliance on host haemoglobin digestion, and due to ROS produced by the host ${ }^{2,4-6}$. Plasmodium apparently lacks some important components of redox systems viz., catalase and a genuine glutathione peroxidase $\mathrm{e}^{5,7}$. It was recently shown that the parasite imports human peroxiredoxin-2 for peroxide detoxification ${ }^{7-11}$. These unique features of Plasmodium redox biology can provide valuable targets for development of specific anti-malarials.

Plasmodium falciparum contains three thioredoxins - Trx1, Trx2, Trx3 and two thioredoxin-like proteins (Tlp1 and Tlp2) $)^{5,12}$. Trx 1 and Tlp1 are cytosolic, Trx3 is localized to the endoplasmic reticulum, while Tlp2 is mitochondrial ${ }^{13}$. Thioredoxin 2 (PfTrx-2 from hereon, MAL13P1.225), the focus of this present work, is a $19 \mathrm{kDa}$ protein with an ER signal sequence. Significantly, PfTrx-2 was recently shown to be part of the so-called translocon complex (PTEX) which is a parasite derived multi-protein machinery resident in parasite parasitophorous vacuolar membrane (PVM), responsible for protein secretion into host cells ${ }^{14}$. Plasmodium parasite remodels the red blood cells by exporting $\sim 5 \%$ of its encoded genome $(\sim 300 \text { proteins })^{15-18}$. These exported proteins are critical for parasite survival in host cells as they play important roles in nutrient uptake, immune evasion and virulence associated functions of the parasite ${ }^{19-22}$. Protein translocation is a complex process as it involves crossing three membranes viz. endoplasmic reticulum, parasite plasma membrane and parasitophorous vacuolar membrane. A hydrophobic signal sequence at the $\mathrm{N}$-terminus of proteins is usually sufficient to target them to the ER and subsequent secretion to the parasitophorous vacuole. However, how proteins are transported out of PVM to the host cytoplasm is not fully understood ${ }^{23-25}$. The situation is even more complex for proteins which are destined for red blood cell plasma membrane. A pentapeptide motif (RxLxE) named Plasmodium export element (PEXEL) has been identified in proteins secreted out of the parasite to the host cell ${ }^{14}$. PEXEL motif is present in both the proteins lacking a signal peptide sequence such as PfEMP1 family as well as those having one like Stevor family proteins ${ }^{25}$. However, many proteins lacking a PEXEL motif are also secreted out by the parasite 
into the host cell by some unknown mechanism. PEXEL motif is a protease recognition site, cleaved by Plasmapsin V in the $\mathrm{ER}^{26,27}$. It is proposed that Plasmapsin $\mathrm{V}$ is more than a signal peptidase and it targets PEXEL containing proteins to the PTEX translocon by channeling the cleaved proteins to HSP101 chaperon ${ }^{25-27}$. It is further hypothesized that at the PTEX proteins are translocated through a membrane pore formed by EXP2 protein ${ }^{14,25}$. Other components of PTEX include PTEX150, PTEX88 and HSP101 which together enable secretion of parasite proteins that contain the PEXEL motif ${ }^{14}$. PfTrx-2 is a component of the PTEX, where it likely plays role of a protein disulfide isomerase, assisting in protein unfolding before translocation ${ }^{14}$. A GFP fused PfTrx-2 was previously shown to be mitochondria localized ${ }^{28}$, but recent studies have shown it to be PVM localized as well as to an organelle of unknown identity, again based on GFP-fusion constructs ${ }^{13,14}$.

Here, we present crystal structure of Plasmodium falciparum Trx2 (PfTrx-2) at $2.9 \AA$ resolution. Evidently, there is only partial conservation in the PfTrx- 2 active site residues, and PfTrx-2 structure reveals several unique features when compared to its human counterpart (HuTrx-2). We also show that disabling PfTrx-2 function by small molecule inhibitors is likely to block or reduce processing/export of hundreds of parasite proteins. This may be a novel strategy to combat parasite growth.

\section{Results}

PfTrx-2 expression, purification, antibody generation and localization. Recombinant PfTrx-2 was very prone to precipitation, and to reduce protein precipitation buffers were maintained in $5 \mathrm{mM}$ DTT while precipitates were pelleted out. The remaining PfTrx-2 in supernatants was found to be fully active (Fig. 1a) and was used for all biochemical and crystallization experiments. PfTrx-2 was

a

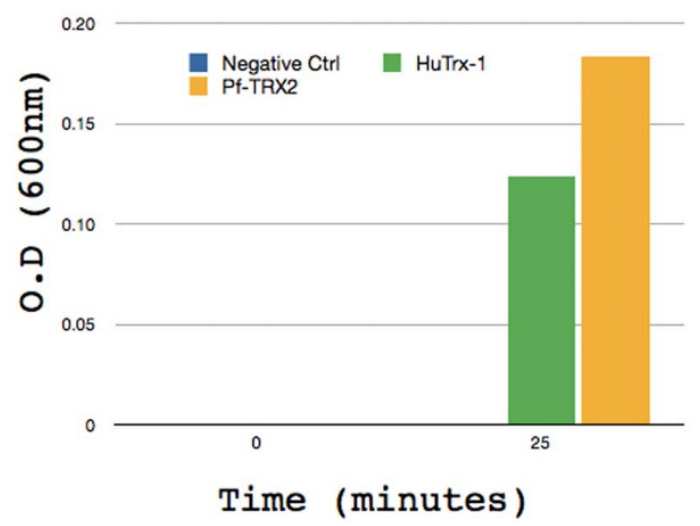

previously shown to be localized to the parasite mitochondrion using GFP-PfTrx 2 constructs ${ }^{28}$. More recently, it was shown to be resident in the parasite PVM and to an unknown parasite organelle ${ }^{13,14}$. We generated antibodies against recombinant PfTrx-2 in rabbit to verify localization of PfTrx-2 at different stages of parasite life cycle. We confirm that PfTrx-2 is localized to the PVM (only) in different asexual stages of Plasmodium (Fig. 2, supplementary movies 1, 2 and 3$)$.

Crystallization and crystal structure of PfTrx-2. Hexagonalshaped crystals were obtained using hanging drop vapor diffusion method by incubating $1 \mu \mathrm{l}$ of protein with $1 \mu \mathrm{l}$ of buffer containing $2.2 \mathrm{M}$ ammonium sulfate and $0.125 \mathrm{mM}$ bis-tris ( $\mathrm{pH} \mathrm{5.5)} \mathrm{at} 20^{\circ} \mathrm{C}$ over a period of $\sim 5$ days. The crystals belong to space group $\mathrm{P} 3{ }_{2} 21$ with two monomers of PfTrx-2 in the asymmetric unit and a solvent content of $\sim 71 \%$. Attempts to solve the crystal structure of PfTrx-2 by molecular replacement failed and PfTrx- 2 was solved by MIRAS techniques using native data along with platinum and mercury derivatives (Table 1, PDB ID 3UL3). Overall structure of PfTrx- 2 consists of four $\beta$ strands $(\beta 1, \beta 2, \beta 3$ and $\beta 4)$, three $\alpha$ helices $(\alpha 1, \alpha 2$ and $\alpha 3)$ and an extended $N$-terminus named L1 (residues 56-71) (Fig. 1b). In PfTrx-2 crystal structure, 25 residues (amino acid numbers 29-53) are disordered as no clear electron density was obvious for them in either $2 \mathrm{Fo}-\mathrm{Fc}$ or Fo-Fc maps. No clear side chain density was observed for residues from 54 to 68 . The overall fold of PfTrx-2 is similar to those of other thioredoxins except for the L1 segment. This extended coil region in PfTrx-2 contacts the other monomer of PfTrx-2 in asymmetric unit (Fig. $3 \mathbf{a}$ and $\mathbf{3 b}$ ). The resulting monomer monomer contacts are not extensive (total surface area per PfTrx-2 is $\sim 5100 \AA^{2}$; buried surface area per PfTrx- $\sim 134.5$

b

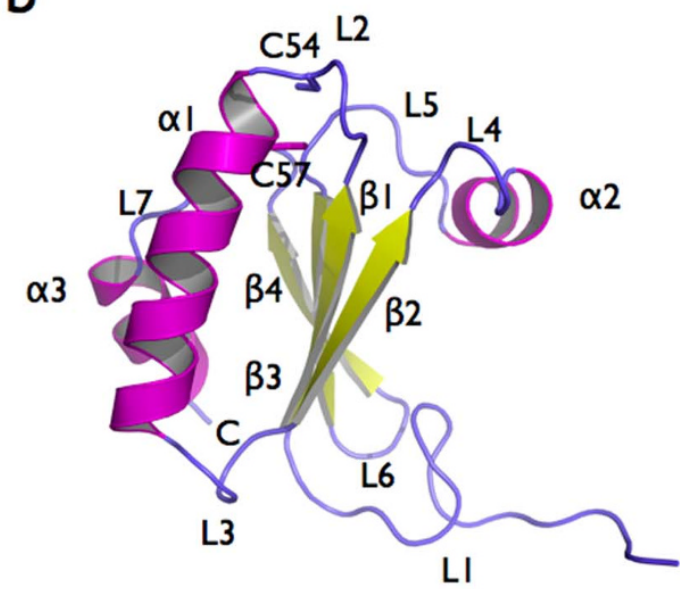

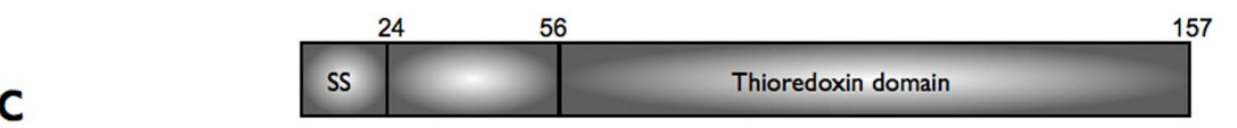
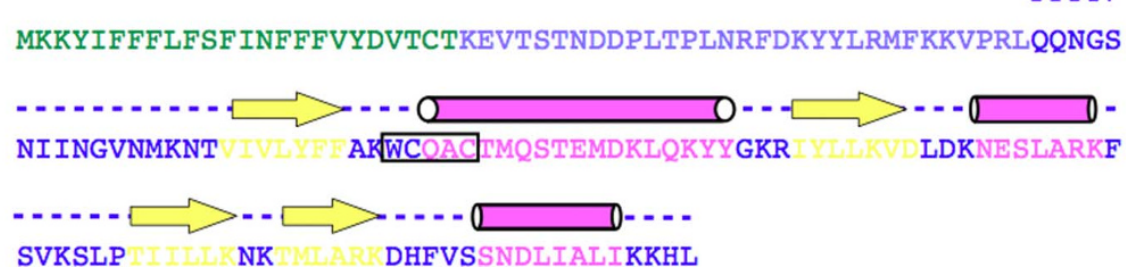

Figure $1 \mid$ PfTrx-2 activity and structure. (a) Insulin reduction assay of PfTrx-2 activity. (b) A cartoon representation of the crystal structure of PfTrx-2. $\alpha$ helices are colored magenta, $\beta$ sheets are colored yellow while $\beta$ turns and loop regions are colored blue. Active site cystines are shown as sticks at the end of $\alpha$ lhelix. (c) A domain diagram of PfTrx-2 representing the structural elements identified in the crystal structure. The color coding is the same as in (b). Residues 1-24 forms the signal sequence region, amino-acids 24-53 are disordered while residues 54-157 form the thioredoxin fold. 


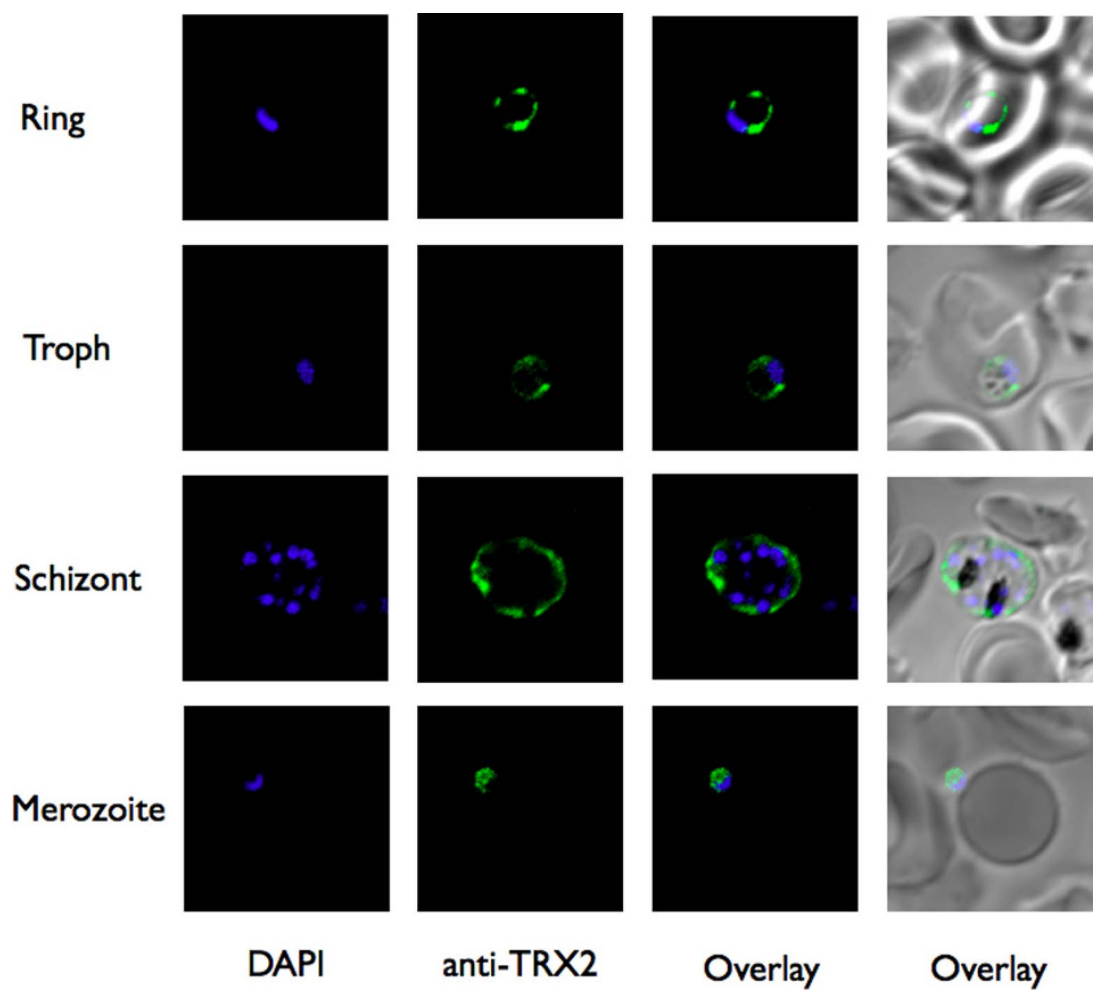

Figure 2 Visible and fluorescence images of 3D7 P. falciparum parasite immunostained with PfTrx-2 antibodies.

$\AA^{2}$ which represents $\sim 2.5 \%$ of the total surface area). L1 is stabilized only partially by interactions with the second PfTrx-2 in the crystal asymmetric unit (Fig. 3a and 3 b). The active site residues of PfTrx-2 (WCQAC) are present at beginning of $\alpha 1$ helix (Fig. 3c). Both molecules of PfTrx-2 are in reduced form with distances between $\mathrm{S}^{\gamma}$ atoms of Cys 82 and Cys 85 of $\sim 3.7 \AA$ and $3.9 \AA$ for $\mathrm{A}$ and $\mathrm{B}$ chains respectively (Fig. $3 \mathrm{c}$ and $3 \mathrm{~d}$ ). Typical distances between sulfurs in disulfide bridges are in $\sim 2 \AA$ range, and a distance of $\sim 3.6 \AA$ represents the minimum distance when a disulfide bridge is absent. We therefore conclude that PfTrx- 2 structure is in a reduced state; despite repeated efforts we were unable to obtain crystals of oxidized PfTrx-2. As expected, PfTrx-2 Cys 85 (pKa $\sim 11)$ is buried inside the protein while Cys $82(\mathrm{pKa} \sim 8)$ is exposed in the loop region at $\alpha 1$ helix terminus. Cys 82 is the active cysteine that likely makes the first nucleophilic attack on substrate disulfides leading to formation intermolecular disulfide bond. This link between PfTrx-2 and its substrate is then likely attacked by Cys 85 which acts as the nucleophile and releases the substrate. The active site Trp 81 is stabilized by an intramolecular hydrogen bond with Asp 111 (Fig. 3d) and is solvent exposed.

Comparison with human mitochondrial thioredoxin. Reduced form of human mitochondrial thioredoxin (PDB code 1UVZ) was superimposed on PfTrx-2 using Chimera ${ }^{29}$. PfTrx-2 shares $\sim 23 \%$ sequence identity with HuTrx-2 (mitochondrial thioredoxin) over its entire length (Fig. 4a). Active site region is only partially conserved (PfTrx-2: WCQAC; Hu-Trx-2: WCGPC) between the two, however their tertiary structures are reasonably conserved (Fig. 4b). The greatest deviation is observed over the $\mathrm{N}$-terminal parts of the structures where the first $\beta$ sheet and first $\alpha$ helix of the human enzyme are absent in case of PfTrx-2 (Fig. 4a). Instead, the Nterminus of PfTrx-2 forms an extended (L1) coiled region (Fig. 4a). When the active site regions of HuTrx-2 and PfTrx-2 were superimposed, we noted several structural variations. Gly 32 and Pro 33 of HuTrx-2 are replaced by Gln 83 and Ala 84 in PfTrx-2. Similarly, the smaller Val 45 in helix $\alpha 2$ ( $\alpha 1$ in PfTrx-2) is replaced by
Gln 96 in PfTrx-2, whose larger side chain would sterically clash with residues in first $\beta$ sheet of HuTrx-2. In HuTrx-2, Glu42 and Thr2 interact to stabilize the first $\beta$ sheet while this is not possible in PfTrx2. Comparision of human and parasite Trx-2 suggests that the first $\alpha$ helix and $\beta$ sheet in PfTrx-2 are displaced relative to HuTrx-2. This distortion in the former results in $\sim 18 \AA$ and $\sim 80^{\circ}$ variation relative to HuTrx-2, exposes PfTrx- $2 \beta$ strands beneath (Fig. $4 a$ ) and creates a groove on the surface of PfTrx-2 (Fig. 4c).

Surface features of PfTrx-2. The electrostatic surface of PfTrx-2 seems positively charged in contrast to the other thioredoxins (including HuTrx-2) that display negatively charged surfaces (Fig. 5a and 5b). Binding of HuTrx-2 with electron acceptor proteins is predicted to involve an acidic cavity near the active site on HuTrx-2 surface - complemented by a basic protuberance due to Lys49 of electron acceptor protein hPDX $5^{30,31-34}$. Residues in this region are conserved in both human and Plasmodium Trx-2 (highlighted by red colored box in Fig. 4b) suggesting retention of binding interfaces for some substrate proteins. However, the L1 region of PfTrx-2 is altogether different from other thioredoxins both electostatically and structurally. A positively charged groove is observed at the L1 region, formed by residues from L1 and from four $\beta$ strands (Fig. 4c). One side of this basic groove is formed by $\alpha 2$ helix while the other is formed by L1 segment. Sequence analysis of other PTEX components shows that two of them viz. PTEX 150 and EXP2 have acidic repeats. Therefore, charge complimentarity between noted regions of PfTrx-2 and PTEX150/EXP2 could result in their engagement with each other (Fig. 4c).

PfTrx-2 Activity inhibition by Aurothiomalate (ATM). ATM is known to inhibit the thioredoxin system ${ }^{35,36}$. Although, ATM was tested for treatment of malaria ${ }^{37,38}$, its mechanism of action is only partially explained by stimulation of eryptosis. Moreover, oxidative stress is known to stimulate eryptosis ${ }^{39}$. Therefore, eryptosis might be an indirect outcome of the action of ATM on thioredoxin system leading to oxidative stress. To investigate this hypothesis, we tested 
Table 1 | X-Ray Data Processing, Structure Solution and Refinement Statistics Values in the parentheses are for the highest resolution shell.

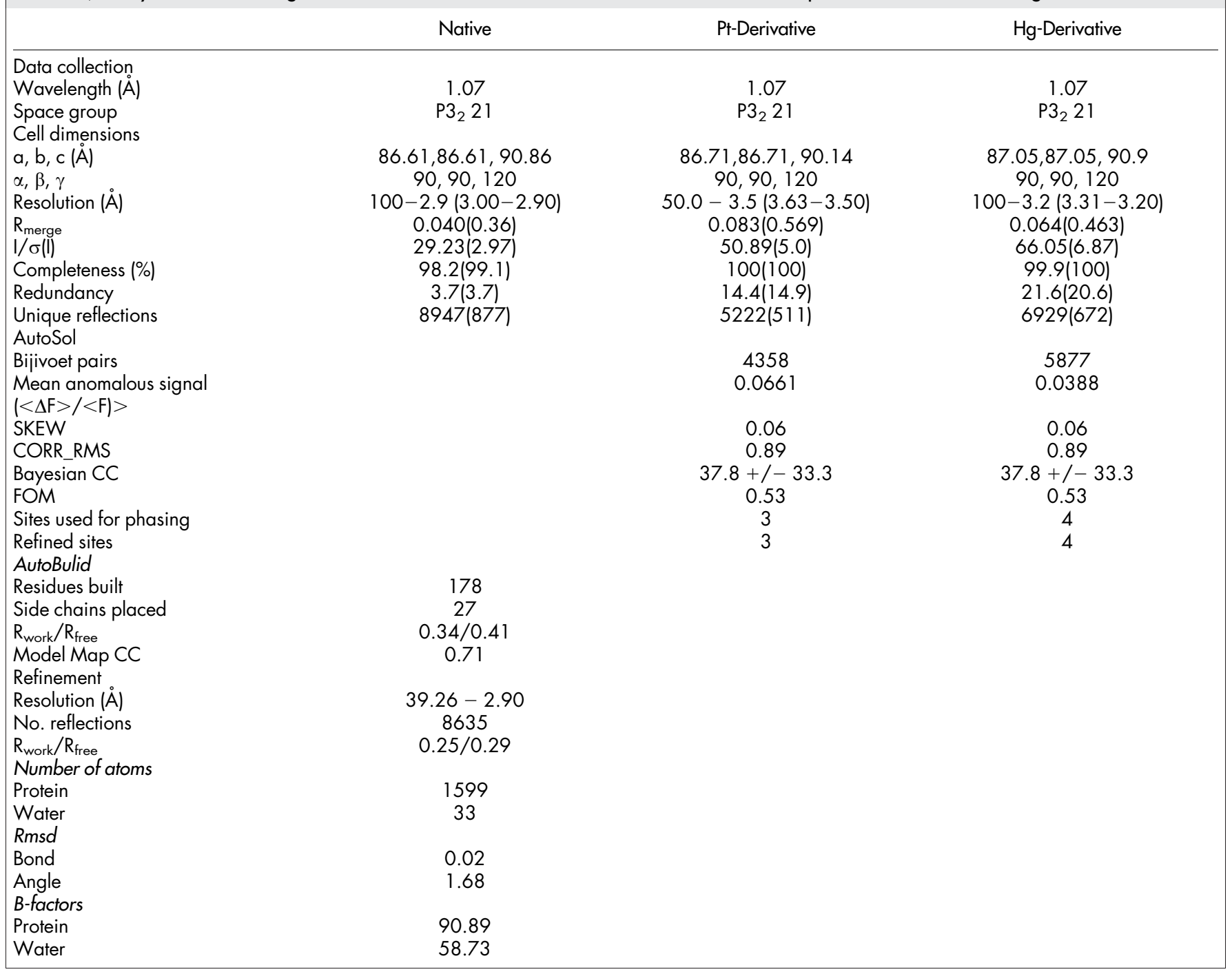

ATM for inhibition of PfTrx-2 activity in an insulin based assay. ATM was able to inhibit the PfTrx-2 activity in a dose dependent manner with an $\mathrm{IC}_{50}$ of $\sim 20 \mu \mathrm{m}$. (Fig. 6a).

In-silico docking of drug-like libraries on PfTrx-2 crystal structure. PfTrx-2 structure shows many unique features which seem useful for selective targeting by drug like compounds. Three regions on the protein surface were selectively targeted - Region 1 , Region 2 and Region 3 (Fig. 7a). Region 1 is the area on the protein surface surrounding the active site. We selected this region as residues in the active site are only partially conserved between humans and Plasmodium (Pf: CQAC, Hu: CGPC). Region 2 is the extended L1 region unique to PfTrx-2, while region 3 is the region on one side of the L1 region (Fig. 7a). Interestingly, top hits docked mostly to L1 (region 2) with a few compounds docking to regions 1 and 3 . We tested top 29 inhibitors which together docked to all three regions on PfTrx-2 surface in parasite viability assays (Fig. 6b). Of the 29 , four inhibitors were able to inhibit parasite growth in micromolar ranges and gave reasonable values for $\mathrm{IC}_{50}$ (Fig. 6c). Intriguingly, the most potent inhibitors from our set docked to the L1 region of PfTrx-2 (Fig. 7c, 7d, 7e and 7f). Inhibitor \# 20, the most effective of those tested, partially shares binding site on PfTrx-2 surface with inhibitor $\# 18$ (Fig. 6b, 7a, 7b and $7 \mathrm{c}$ and $7 \mathrm{e}$ ). Inhibitor \#28 docked to region 3 of PfTrx-2 (Fig. 7a and 7f), while inhibitor \#19 docked within the L1 region but distal from where inhibitor 18 and 20 docked (Fig. 7a and 7d). Despite having different skeletal types (the SPECS diversity set library was used), these inhibitors seem to make similar interactions with residues on surface of PfTrx-2. They therefore can serve as chemical starting points for development of more specific and potent Trx 2 inhibitors.

\section{Discussion}

Malaria parasites have evolved clever strategies for survival in multiple hosts. One such facet to malaria parasites is their ability to extensively remodel host cells by exporting numerous parasite proteins ${ }^{15-18}$. PfTrx-2 is a component of the translocon of exported proteins (PTEX), located on the parasite PVM where it purportedly helps in protein folding/unfolding before substrate secretion. We recombinantly expressed PfTrx-2 in E. coli and purified the protein to homogeneity by conventional chromatographic techniques. Protein activity was tested by an insulin reduction assay which suggested enzymatically competent PfTrx-2 (Fig. 1a). In agreement with earlier studies, we confirmed PVM localization of PfTrx-2 using polyclonal antibodies specifically generated against recombinant PfTrx-2 (Fig. 2). We could not confirm localization of PfTrx-2 to any organelle of unknown identity inside the parasite as has been reported earlier ${ }^{13}$. Confocal imagery-based movies which show distinct localization of PfTrx-2 in the erythrocytic stages accompany this manuscript (supplementary data). 


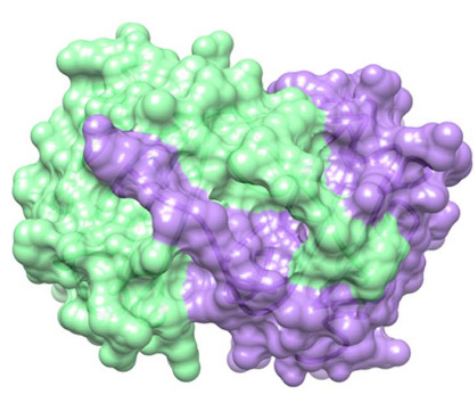

a

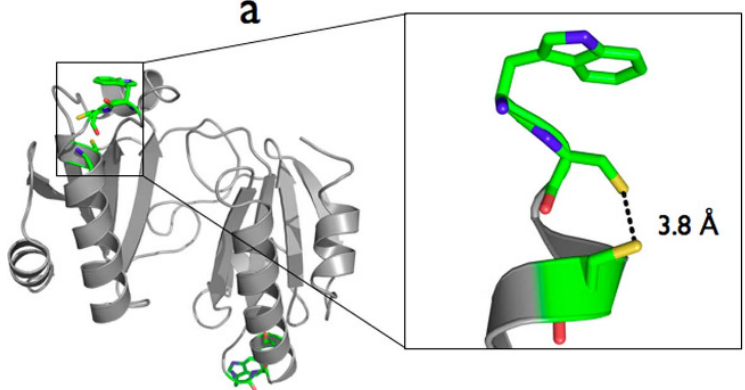

C

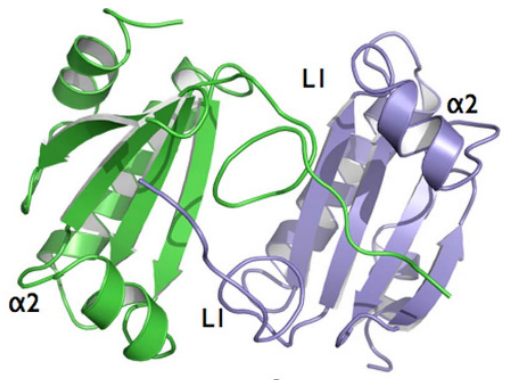

b

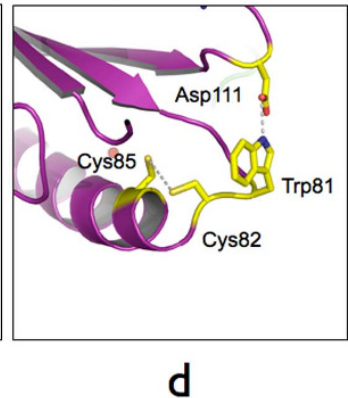

Figure 3 Two molecules of PfTrx-2 in the crystallographic asymmetric unit. (a) A surface representation of PfTrx-2. (b) A cartoon representation showing L1 regions of the two PfTrx-2 contacting each other. (c) Active site of PfTrx-2 showing two cysteines in a reduced state and (d) Trp 81 interacts with the Asp 111 through a hydrogen bond.

Aurothiomalate is a known inhibitor of thioredoxin reductase in $\mathrm{rat}^{36}$ and is used for the treatment of rheumatoid arthritis and cancer $^{37}$. Interestingly, aurothiomalate also reduces Plasmodium parasitemia both in vitro and in infected mice ${ }^{38}$. However, the mechanism of anti-parasitic action for aurothiomalate could only be partially explained - entailing stimulation of eryptosis in infected RBCs ${ }^{38}$. a

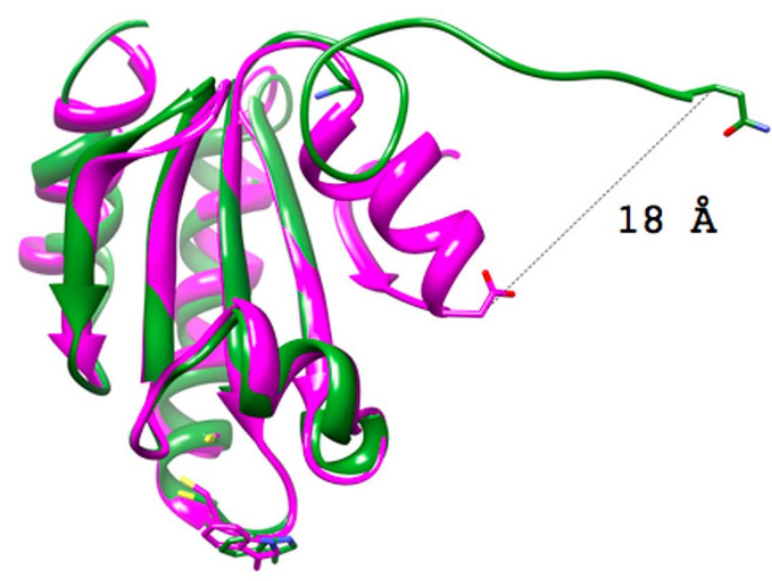

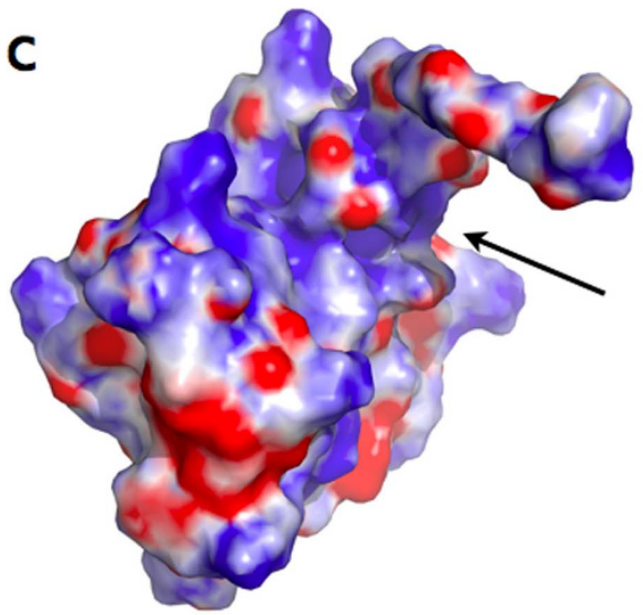

b

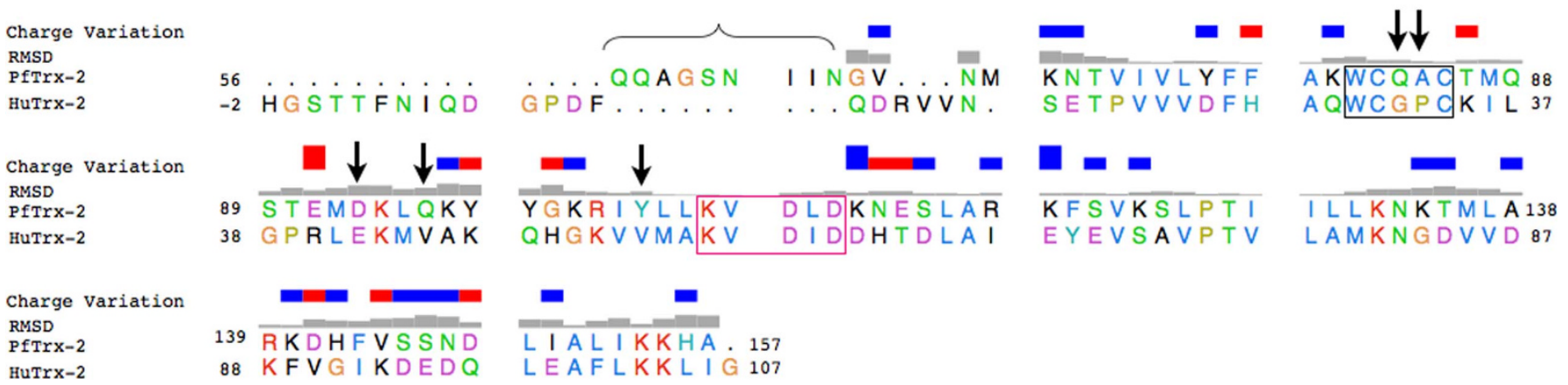

Figure $4 \mid$ Comparision of human and $\boldsymbol{P}$. falciparum thioredoxin structures. (a) A structural superposition of Pftrx-2 (green) and HuTrx-2 (magenta). L1 region is displaced by $\sim 18 \AA$ and $\sim 80$ degrees in PfTrx-2. (b) A structure based sequence alignment of PfTrx-2 and HuTrx-2. (c) Electrostatic surface representation of PfTrx-2. A basic groove (marked by black arrow) is evident on the surface of PfTrx-2 where acidic regions of PTEX proteins can dock. 
a
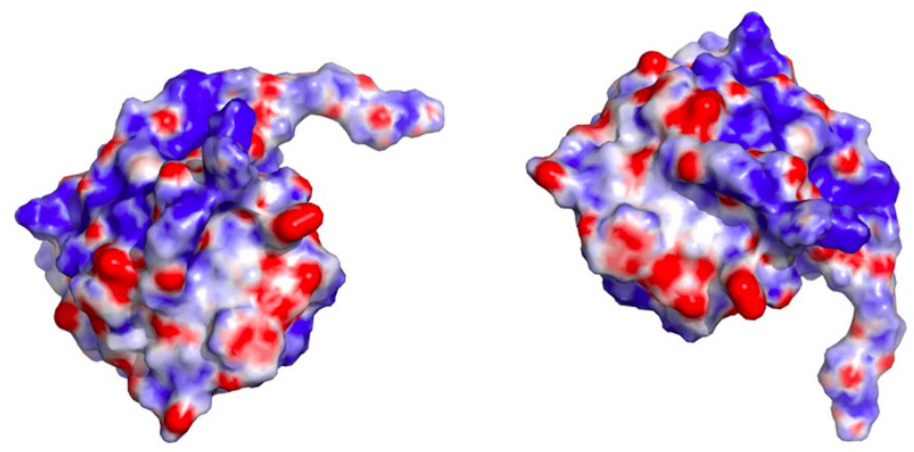

b
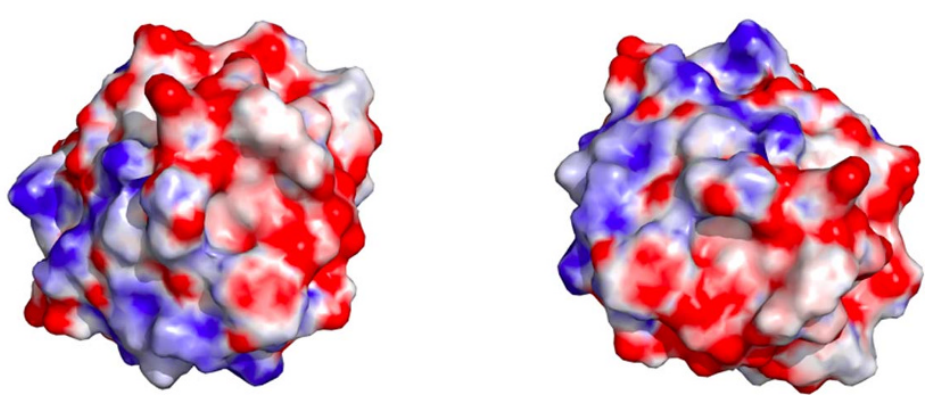

Figure 5 Comparison of surface electrostatics of (a) PfTrx-2 and (b) HuTrx-2. Each view is rotated by 180 degrees with respect to the previous view.

Here, we show that aurothiomalate inhibits PfTrx-2 activity in a dose dependent manner with an $\mathrm{IC}_{50}$ of $\sim 20 \mu \mathrm{M}$ (Fig. 6a). The growth inhibitory effects of aurothiomalate might therefore be due to blockage of protein secretion via the translocon where PfTrx-2 resides. Besides three thioredoxins and two thioredoxin-like proteins, Plasmodium falciparum additionally encodes a thioredoxin reductase
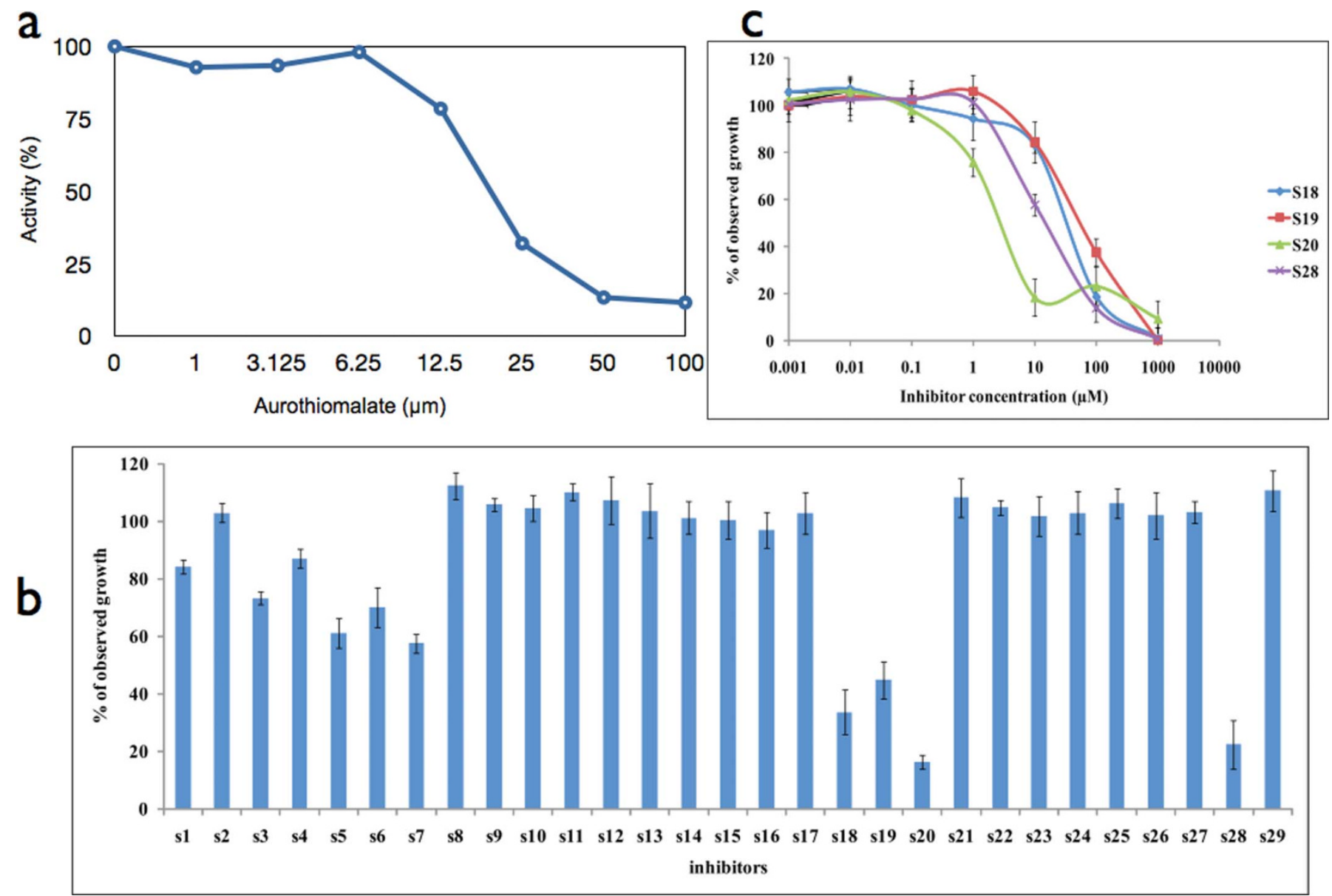

Figure $6 \mid$ PfTrx-2 inhibition assays. (a) Aurothiomalate inhibition assay. (b) Parasite growth inhibition assay (PIA) performed using $100 \mu \mathrm{M}$ of different inhibitors. (c) PIA performed with four best inhibitors selected from (b) using a concentration range from $0.001 \mu \mathrm{M}$ to $10000 \mu \mathrm{M}$. 


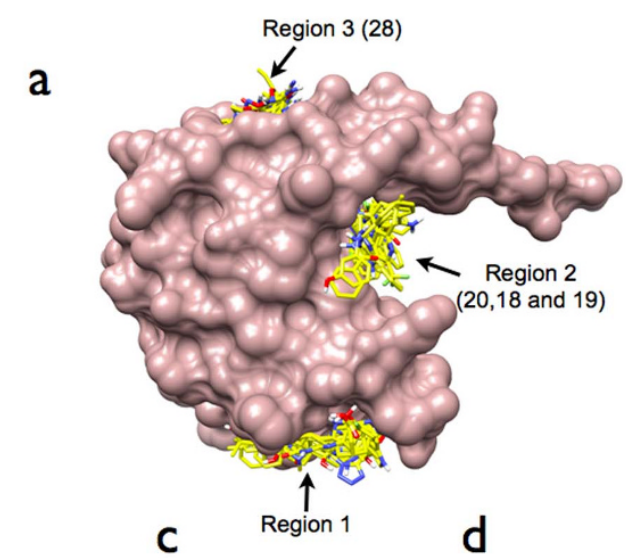

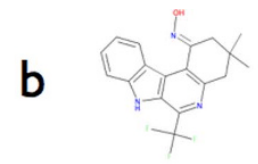

18

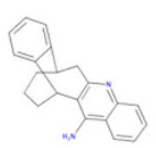

20

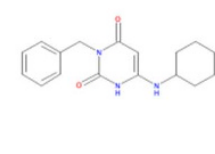

19

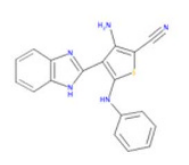

28
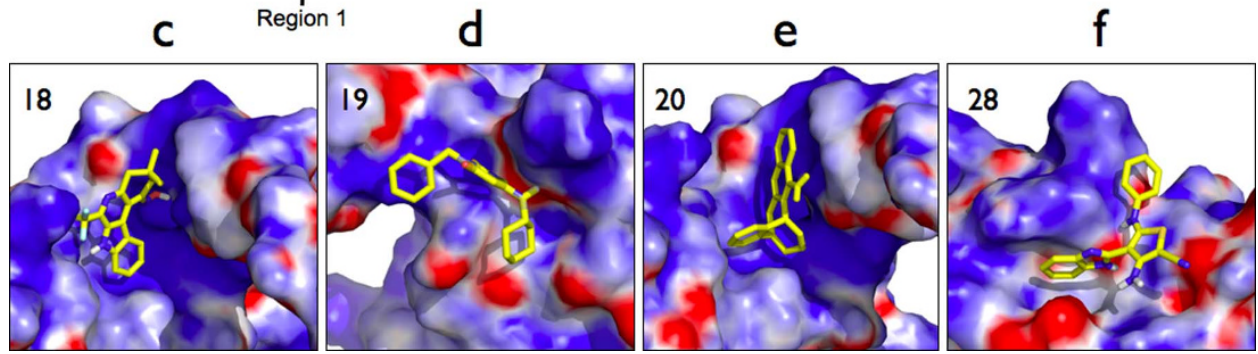

Figure 7 | In-silico docking of inhibitors on PfTrx-2. (a) Three regions selected for docking analysis showing the 29 inhibitors selected for parasite growth inhibition assay (PIA). (b) Molecular structure of top four inhibitors (Inhibitor number 18, 19, 20, and 28, respectively) (18) C18H16F3N3O (3,3-dimethyl6-(trifluoromethyl)-2,3,4,7-tetrahydro-1H-indolo[2,3-c] quinolin-1-one oxime) (19) C17H21N3O2 (3-benzyl-6-(cyclohexylamino)-2,4(1H,3H)pyrimidinedione)(20) C21H20N2 (10-azapentacyclo[11.6.3.0 2,11 .0 4,9 .0 14,19 ] docosa-2(11),3,5,7,9,14,16,18-octaen-3-ylamine) and (28) C18H13N5S (3-amino-5-anilino-4-(1H-benzimidazol-2-yl)thiophene-2-carbonitrile). (c)-(f) Docking of top four inhibitors from PIA on PfTrx-2 surface.

which shows a dual localization to both cytoplasm and mitochondrion of the Plasmodium parasites ${ }^{13}$. One or several of these parasite proteins could therefore be the target of aurothiomalate action.

Structural analysis of PfTrx-2 highlights unique features in the Plasmodium enzyme when compared to its human counterpart (Fig. 1b). The N-terminal $\alpha$ helix and $\beta$ strand in HuTrx-2 are replaced by an extended coil (L1 region) in PfTrx- 2 structure. L1 is stabilized by interactions with another PfTrx- 2 protomer in the crystal asymmetric unit. This L1 region of PfTrx-2 is largely basic in nature, and could potentially form interaction interfaces for the numerous acidic regions in PTEX150 and EXP2 (Fig. 4c). The strikingly different electrostatic surface properties of Pf Trx-2 and HuTrx2 suggest different modes of interaction, with possibly different protein partners (Fig. 5). Opposite locations for PfTrx-2 active site and its $L 1$ region suggests that the spatial segregation may be biologically relevant. For example, one outcome of distal locations of PfTrx2 active site and L1 region may be to allow simultaneous engagement of PfTrx 2 with PTEX components on one side and substrates on the other side. This unique architectural feature therefore presents opportunities for targeting PfTrx-2 with small molecular inhibitors directed against one or both sites. Our crystallographic analysis therefore supports targeting PfTrx-2 for structure guided drug design. Interestingly, our in silico docking studies with drug-like libraries show that most of the top docking poses localize within the L1 region of PfTrx-2 (region 2 in Fig. 6b, Fig. 7a). In summary, our structural-functional and inhibitor discovery data together provide a platform for development of more potent PfTrx-2 inhibitors that can impede protein export from malaria parasites by interfering with the interaction interfaces of PfTrx-2.

\section{Methods}

Molecular cloning, Expression and Purification. Pf Trx-2 gene contains a predicted ER signal sequence at the N-terminus. Gene construct of PfTrx-2, without its signal sequence from amino acids 28-157, was designed for optimal expression in E. coli. The construct was sub-cloned in pGEX4T1 vector with BamH1 and Sall restriction sites and protein was expressed as a GST fusion. For overproduction of the fused protein, Escherhichia coli Lemo21 DE3 competent cells were freshly transformed, and a single colony was grown in $10 \mathrm{ml}$ of LB media containing $100 \mu \mathrm{g} / \mathrm{ml}$ ampicillin. After 12 hours of growth, this seed culture was used to inoculate 1 liter of LB media that was grown for 3.5 hours at $37^{\circ} \mathrm{C}$. Protein expression was induced by adding $0.25 \mathrm{mM}$ isopropyl $\beta$-D-thioglactopyranoside, and the culture was grown for 20 hours post-induction at $16^{\circ} \mathrm{C}$. Cells were harvested by centrifugation at $5000 \mathrm{X} \mathrm{g}$ for 15 minutes. The resulting bacterial pellet was suspended in a buffer containing 1XPBS, $5 \mathrm{mM}$ DTT and protease inhibitor cocktail (Roche). Cells were lysed by sonication and cleared by centrifugation at 20,000 X $\mathrm{g}$ for 45 minutes. The cleared supernatant was applied to $5 \mathrm{ml}$ GST FF column (GE Biosciences). The GST-Trx2 fusion protein was purified by elution with a buffer containing 1XPBS, $5 \mathrm{mM}$ DTT and $20 \mathrm{mM}$ reduced glutathione on AKTA automated protein purifier (GE Biosciences). The purified protein was desalted in 1X PBS containing $5 \mathrm{mM}$ DTT using a desalting column and the GST tag was removed by incubating with thrombin protease in 1XPBS (GE Biosciences) at $20^{\circ} \mathrm{C}$ overnight. The cleaved protein was concentrated by using $3 \mathrm{KDa}$ cutoff centricon centrifugal device (Millipore) and purified by gel filtration chromatography on S75 gel-filtration column (GE Biosciences). Best fractions were checked by SDS-PAGE and those containing pure PfTrx-2 were pooled and buffer-exchanged in $20 \mathrm{mM}$ Tris $\mathrm{pH} 8.0,20 \mathrm{mM} \mathrm{NaCl}$ and $10 \mathrm{mM}$ DTT. The protein was concentrated to $5 \mathrm{mg} / \mathrm{ml}$ using a $3 \mathrm{KDa}$ centricon centrifugal device (Millipore) and stored at $-80^{\circ} \mathrm{C}$.

Immunofluorescence assays using confocal microscopy. Anti-PfTrx-2 antibodies raised in a rabbit were used in immunofluorescence assays for localization of native PfTrx-2 within malaria parasites. The assay was performed as described earlier ${ }^{40}$. Cells were washed in PBS and fixed in solution using $4 \%$ paraformaldehyde and $0.0075 \%$ glutaraldehyde in PBS for 25 minutes. After a PBS wash, cells were permeabilized by using $0.1 \%$ Triton X-100 in PBS for 10 minutes. After another PBS wash, cells were treated with $0.1 \mathrm{mg} / \mathrm{ml}$ sodium borohydride in PBS for 10 minutes. Cells were washed again with PBS, blocked in 3\% BSA/PBS for 1 hour and incubated overnight with protein-A column purified rabbit anti-protein IgG antibody (1:250 dilution) at $4{ }^{\circ} \mathrm{C}$. Cells were washed three times with PBS for 10 minutes each and incubated with Alexaflour 488-tagged anti-rabbit secondary antibody for 1 hour at room temperature and allowed to settle onto coverslips coated with poly-L-lysine $(100 \mathrm{mg} / \mathrm{ml})$. Finally, the coverslips were washed three times in PBS, mounted in antifade with DAPI reagent (Invitrogen) and sealed. Confocal microscopy was performed on a Nikon eclipse TE2000U microscope. Pre-immune sera were taken as negative controls.

Crystallization, Data Collection and Structure Determination. PfTrx-2 crystals were obtained at $20^{\circ} \mathrm{C}$ by hanging drop vapor diffusion method using $1 \mu \mathrm{l}$ of PfTrx-2 $(5 \mathrm{mg} / \mathrm{ml})$ and $1 \mu \mathrm{l}$ of $0.125 \mathrm{M}$ Bis-tris pH 5.5, $2.2 \mathrm{M}$ ammonium sulfate and $5 \mathrm{mM}$ DTT. Single, hexagonal shaped crystals were added to a cryoprotectant solution containing $0.125 \mathrm{mM}$ Bis-tris pH 5.5, 3.0 M ammonium sulfate and $20 \%$ glycerol for 1 minute before flash freezing in cooled nitrogen gas at $100 \mathrm{~K}$. For phasing, crystals were soaked in $100 \mathrm{mM}$ mercury (II) potassium iodide in cryoprotectant solution 
before flash freezing. For Pt derivatives, crystals were soaked in $100 \mathrm{mM}$ Di- $\mu$ idobis(ethylenediamine) diplatinum (II) nitrate in cryoprotectant solution before flash freezing. X-ray diffraction data were collected on a MARCCD detector at BM14 beam line of European Synchrotron Radiation Facility at Grenoble, France. Pt(II) and $\mathrm{Hg}$ (II) derivative datasets were collected along with native data in frozen conditions. The diffraction images were processed and scaled with $H K L 2000$ suite program $^{41}$. Four Hg and three Pt sites were found by AutoSol in Phenix and by SHELXD ${ }^{42,43}$. PfTrx- 2 crystals are trigonal and belong to the space group $\mathrm{P}_{2} 21$ (Table 1). The structure was solved by MIRAS procedures using native and derivative datasets in PHENIX ${ }^{42}$. Initial model was build by AutoBuild in PHENIX, which was subsequently rebuilt manually using $\mathrm{COOT}^{44}$. Model refinement was performed using phenix.refine in PHENIX ${ }^{42}$.

Activity assay and Inhibition of PfTrx-2 activity. Enzymatic activity of recombinant $\mathrm{Pf}$ Trx- 2 was determined by insulin reduction assay ${ }^{45}$. Briefly, $2.0-8.0$ $\mu \mathrm{g}$ of recombinant $\mathrm{Pf}$ Trx- 2 was used in $1 \mathrm{ml}$ of $0.1 \mathrm{M}$ potassium phosphate ( $\mathrm{pH} 7.0$ ), $2 \mathrm{mM}$ EDTA, $0.1 \%$ bovine insulin, and $1 \mathrm{mM}$ DTT. The same buffer without DTT was used as reference. Protein concentration was determined by using the Bio-Rad protein assay kit with BSA as standard. Thioredoxin activity was measured at $25^{\circ} \mathrm{C}$ and defined as the maximal increase rate of turbidity at O.D. at $650 \mathrm{~nm}$ due to insulin precipitation $^{45}$. For inhibition of Pf Trx- 2 activity, varying amounts of inhibitors were added in the reaction mixtures and decrease in turbidity at O.D. $650 \mathrm{~nm}$ due to inhibition of insulin precipitation was measured.

In silico screening of PfTrx-2 inhibitors. Molecular docking and in silico screening were carried using PfTrx-2 structure in docking program AutoDock vina ${ }^{46}$ with a SPECS “Specs world diversity” library. The library was prepared using Ligprep module in Schrodinger software suite to ensure proper partial charges and protonation states. Physiochemical properties were predicted using Qikprop module in Schrödinger suite. For in silico screening, we applied 10 lead-like filters derived from Lipinski's rule of five: $1<\mathrm{QPlog} \mathrm{P}_{\mathrm{o} / \mathrm{w}}<4 ; 100<$ molecular weight $<350$; $\mathrm{H}$ donor $<5$; $\mathrm{H}$ acceptor $<$ 10; rotatable bounds $<15 ;-5<$ QPlogS $>-1$; $300 \AA<$ SASA $>1000 \AA ; 0 \AA<$ FOSA $>750 \AA ; 7 \AA<$ FISA > $330 \AA$, FISA > FOSA. After an initial lead-like filtering process, 900 compounds were collated for screening. The top docking poses were isolated from each output file for further analysis. Top ligands were selected based on docking scores and H-bond interactions with residues in PfTrx-2.

Parasite Growth Inhibition Assays. Top inhibitors selected via above procedure were tested on malaria parasite $P$. falciparum 3D7 strain. Parasites were synchronized using sorbitol and starting with ring stages these parasites were cultured in 96 well plates at $1 \%$ parasitemia and $2 \%$ hematocrit. Initial screening of all top compounds was performed by growing parasites at $100 \mu \mathrm{M}$ inhibitor concentration. After 48 hours of growth, parasitemia was measured by fluorescence assay as described earlier ${ }^{47}$. Briefly, culture was lysed by adding $100 \mu \mathrm{l}$ of lysis buffer [Tris $(20 \mathrm{mM}, \mathrm{pH}$ 7.5), EDTA (5 mM), saponin $(0.008 \%$, w/v) and Triton X-100(0.08\%, w/v)], SYBR green I was added to each well $(0.2 \mu \mathrm{l}$ of SYBR Green in $1 \mathrm{ml}$ of lysis buffer), and mixed until no erythrocyte sediments were visible. After 1 hour incubation in dark at room temperature, fluorescence was measured with multi-well plate reader (Victor3, Perkin Elmer) at excitation and emission wavelength bands centered at $485 \mathrm{~nm}$ and $530 \mathrm{~nm}$, respectively. To access the effect of selected inhibitors, fluorescence readings were compared and subtracted from fluorescence values at $100 \%$ parasite inhibition fluorescence value at $100 \mu \mathrm{M}$ chloroquine was taken as $100 \%$ parasite growth inhibition. We then calculated percentage parasitemia for each inhibitor. To further validate the anti-malarial activity of top compounds from this first screen, experiments were repeated with a broader range of inhibitor concentrations ranging from $1 \mathrm{nM}$ to $1 \mathrm{mM}$. Each experiment was repeated three times and each value is average of four measurements. These data were used to calculate $\mathrm{IC}_{50}$ values for each inhibitor.

1. WHO. World malaria report. http://www.who.int/mediacentre/factsheets/fs094/ en/index.html (2009)

2. Becker, K. et al. Oxidative stress in malaria parasite-infected erythrocytes: hostparasite interactions. Int J Parasitol 34, 163-89 (2004).

3. Wajcman, H. \& Galacteros, F. [Glucose 6-phosphate dehydrogenase deficiency: a protection against malaria and a risk for hemolytic accidents]. C R Biol 327, 711-20 (2004)

4. Bozdech, Z. \& Ginsburg, H. Antioxidant defense in Plasmodium falciparum--data mining of the transcriptome. Malaria Journal 3, 23 (2004).

5. Muller, S. Redox and antioxidant systems of the malaria parasite Plasmodium falciparum. Mol Microbiol 53, 1291-305 (2004).

6. Liochev, S. I. \& Fridovich, I. Superoxide and iron: partners in crime. IUBMB Life 48, 157-61 (1999).

7. Koncarevic, S. et al. The malarial parasite Plasmodium falciparum imports the human protein peroxiredoxin 2 for peroxide detoxification. Proc Natl Acad Sci U S A 106, 13323-8 (2009).

8. Kanzok, S. M., Schirmer, R. H., Turbachova, I., Iozef, R. \& Becker, K. The thioredoxin system of the malaria parasite Plasmodium falciparum. Glutathione reduction revisited. J Biol Chem 275, 40180-6 (2000).

9. Rahlfs, S., Schirmer, R. H. \& Becker, K. The thioredoxin system of Plasmodium falciparum and other parasites. Cell Mol Life Sci 59, 1024-41 (2002).

10. Becker, K., Rahlfs, S., Nickel, C. \& Schirmer, R. H. Glutathione--functions and metabolism in the malarial parasite Plasmodium falciparum. Biol Chem 384, 55166 (2003).
11. Muller, S. Thioredoxin reductase and glutathione synthesis in Plasmodium falciparum. Redox Rep 8, 251-5 (2003).

12. Nickel, C., Rahlfs, S., Deponte, M., Koncarevic, S. \& Becker, K. Thioredoxin networks in the malarial parasite Plasmodium falciparum. Antioxid Redox Signal 8, 1227-39 (2006).

13. Kehr, S., Sturm, N., Rahlfs, S., Przyborski, J. M. \& Becker, K. Compartmentation of redox metabolism in malaria parasites. PLoS Pathog 6, e1001242 (2010).

14. de Koning-Ward, T. F. et al. A newly discovered protein export machine in malaria parasites. Nature 459, 945-9 (2009).

15. Marti, M., Good, R. T., Rug, M., Knuepfer, E. \& Cowman, A. F. Targeting malaria virulence and remodeling proteins to the host erythrocyte. Science 306, 1930-3 (2004).

16. Hiller, N. L. et al. A host-targeting signal in virulence proteins reveals a secretome in malarial infection. Science 306, 1934-7 (2004).

17. Sargeant, T. J. et al. Lineage-specific expansion of proteins exported to erythrocytes in malaria parasites. Genome Biol 7, R12 (2006).

18. van Ooij, C. et al. The malaria secretome: from algorithms to essential function in blood stage infection. PLoS Pathog 4, e1000084 (2008)

19. Crabb, B. S. et al. Targeted gene disruption shows that knobs enable malariainfected red cells to cytoadhere under physiological shear stress. Cell 89, 287-96 (1997)

20. Waterkeyn, J. G. et al. Targeted mutagenesis of Plasmodium falciparum erythrocyte membrane protein 3 (PfEMP3) disrupts cytoadherence of malariainfected red blood cells. EMBO J 19, 2813-23 (2000).

21. Glenister, F. K., Coppel, R. L., Cowman, A. F., Mohandas, N. \& Cooke, B. M. Contribution of parasite proteins to altered mechanical properties of malariainfected red blood cells. Blood 99, 1060-3 (2002).

22. Maier, A. G. et al. Exported proteins required for virulence and rigidity of Plasmodium falciparum-infected human erythrocytes. Cell 134, 48-61 (2008).

23. Tonkin, C. J., Pearce, J. A., McFadden, G. I. \& Cowman, A. F. Protein targeting to destinations of the secretory pathway in the malaria parasite Plasmodium falciparum. Curr Opin Microbiol 9, 381-7 (2006).

24. Klemba, M., Beatty, W., Gluzman, I. \& Goldberg, D. E. Trafficking of plasmepsin II to the food vacuole of the malaria parasite Plasmodium falciparum. J Cell Biol 164, 47-56 (2004).

25. Goldberg, D. E. \& Cowman, A. F. Moving in and renovating: exporting proteins from Plasmodium into host erythrocytes. Nat Rev Microbiol 8, 617-21 (2010).

26 . Boddey, J. A. et al. An aspartyl protease directs malaria effector proteins to the host cell. Nature 463, 627-31 (2010).

27. Russo, I. et al. Plasmepsin V licenses Plasmodium proteins for export into the host erythrocyte. Nature 463, 632-6 (2010)

28. Boucher, I. W. et al. Structural and biochemical characterization of a mitochondrial peroxiredoxin from Plasmodium falciparum. Mol Microbiol 61, 948-59 (2006).

29. Pettersen, E. F. et al. UCSF Chimera--a visualization system for exploratory research and analysis. J Comput Chem 25, 1605-12 (2004).

30. Smeets, A. et al. Crystal structures of oxidized and reduced forms of human mitochondrial thioredoxin 2. Protein Sci 14, 2610-21 (2005).

31. Lennon, B. W., Williams, C. H., Jr. \& Ludwig, M. L. Twists in catalysis: alternating conformations of Escherichia coli thioredoxin reductase. Science 289, 1190-4 (2000)

32. Sandalova, T., Zhong, L., Lindqvist, Y., Holmgren, A. \& Schneider, G. Threedimensional structure of a mammalian thioredoxin reductase: implications for mechanism and evolution of a selenocysteine-dependent enzyme. Proc Natl Acad Sci U S A 98, 9533-8 (2001).

33. Katti, S. K., LeMaster, D. M. \& Eklund, H. Crystal structure of thioredoxin from Escherichia coli at 1.68 A resolution. J Mol Biol 212, 167-84 (1990).

34. Capitani, G. et al. Crystal structures of two functionally different thioredoxins in spinach chloroplasts. J Mol Biol 302, 135-54 (2000).

35. Sannella, A. R. et al. New uses for old drugs. Auranofin, a clinically established antiarthritic metallodrug, exhibits potent antimalarial effects in vitro: Mechanistic and pharmacological implications. FEBS Lett 582, 844-7 (2008).

36. Rigobello, M. P., Scutari, G., Folda, A. \& Bindoli, A. Mitochondrial thioredoxin reductase inhibition by gold(I) compounds and concurrent stimulation of permeability transition and release of cytochrome c. Biochemical Pharmacology 67, 689-696 (2004)

37. Shaw, C. F. Gold-Based Therapeutic Agents. Chem. Rev. 99 2589-2600. (1999)

38. Alesutan, I. et al. Beneficial effect of aurothiomalate on murine malaria. Malar J9, 118 (2010).

39. Tyurina, Y. Y. et al. Oxidation of phosphatidylserine: a mechanism for plasma membrane phospholipid scrambling during apoptosis? Biochem Biophys Res Commun 324, 1059-64 (2004).

40. Stowers, A. W. et al. Efficacy of two alternate vaccines based on Plasmodium falciparum merozoite surface protein 1 in an Aotus challenge trial. Infect Immun 69, 1536-46 (2001)

41. Otwinowski, Z. \& Minor, W. [20] Processing of X-ray diffraction data collected in oscillation mode. in Methods in Enzymology, Vol. Volume 276 (ed. Charles, W. Carter, Jr.) 307-326 (Academic Press, 1997).

42. Adams, P. D. et al. PHENIX: a comprehensive Python-based system for macromolecular structure solution. Acta Crystallographica Section D 66, 213-221 (2010).

43. Schneider, T. R. \& Sheldrick, G. M. Substructure solution with SHELXD. Acta Crystallogr D Biol Crystallogr 58, 1772-9 (2002). 
44. Emsley, P. \& Cowtan, K. Coot: model-building tools for molecular graphics. Acta Crystallogr D Biol Crystallogr 60, 2126-32 (2004).

45. Holmgren, A. Thioredoxin catalyzes the reduction of insulin disulfides by dithiothreitol and dihydrolipoamide. J Biol Chem 254, 9627-32 (1979).

46. Trott, O. \& Olson, A. J. AutoDock Vina: Improving the speed and accuracy of docking with a new scoring function, efficient optimization, and multithreading. Journal of Computational Chemistry 31, 455-461 (2010).

47. Smilkstein, M., Sriwilaijaroen, N., Kelly, J. X., Wilairat, P. \& Riscoe, M. Simple and inexpensive fluorescence-based technique for high-throughput antimalarial drug screening. Antimicrob Agents Chemother 48, 1803-6 (2004).

\section{Acknowledgements}

The X-ray facility at ICGEB is funded by Wellcome Trust, UK. Ashwani Sharma is supported by University Grants Commission, Government of India. Amit Sharma laboratory is supported by grants from EU, DBT (Govt of India) and the Wellcome Trust, UK.

\section{Author contributions}

Ashwani Sharma purified, crystallized and solved Pf Trx-2 structure. Sameer Dixit assisted with protein work while Arvind Sharma performed confocal studies and parasite growth assays. Ashwani Sharma and Amit Sharma conceived the study, designed all experiments and wrote the manuscript.

\section{Additional information}

Supplementary information accompanies this paper at http://www.nature.com/ scientificreports

Competing financial interests: The authors declare no competing financial interests.

License: This work is licensed under a Creative Commons

Attribution-NonCommercial-ShareAlike 3.0 Unported License. To view a copy of this license, visit http://creativecommons.org/licenses/by-nc-sa/3.0/

How to cite this article: Sharma, A., Sharma, A., Dixit, S. \& Sharma, A. Structural insights into thioredoxin-2: a component of malaria parasite protein secretion machinery. Sci. Rep. 1, 179; DOI:10.1038/srep00179 (2011). 